\title{
Some Trapezoidal Vector Inequalities for Continuous Functions of Selfadjoint Operators in Hilbert Spaces
}

\author{
S. S. Dragomir ${ }^{1,2}$ \\ ${ }^{1}$ Mathematics, School of Engineering Science, Victoria University, P.O. Box 14428, Melbourne City, \\ VIC 8001, Australia \\ ${ }^{2}$ School of Computational Applied Mathematics, University of the Witwatersrand, \\ Private Bag 3, Johannesburg 2050, South Africa
}

Correspondence should be addressed to S. S. Dragomir, sever.dragomir@vu.edu.au

Received 24 November 2010; Accepted 31 March 2011

Academic Editor: Ferhan M. Atici

Copyright (C 2011 S. S. Dragomir. This is an open access article distributed under the Creative Commons Attribution License, which permits unrestricted use, distribution, and reproduction in any medium, provided the original work is properly cited.

On utilising the spectral representation of selfadjoint operators in Hilbert spaces, some trapezoidal inequalities for various classes of continuous functions of such operators are given.

\section{Introduction}

In classical analysis a trapezoidal type inequality is an inequality that provides upper and/or lower bounds for the quantity

$$
\frac{f(a)+f(b)}{2}(b-a)-\int_{a}^{b} f(t) d t
$$

that is, the error in approximating the integral by a trapezoidal rule, for various classes of integrable functions $f$ defined on the compact interval $[a, b]$.

In order to introduce the reader to some of the well-known results and prepare the background for considering a similar problem for functions of selfadjoint operators in Hilbert spaces, we mention the following inequalities.

The case of functions of bounded variation was obtained in [1] (see also [1, p. 68]):

Theorem 1.1. Let $f:[a, b] \rightarrow \mathbb{C}$ be a function of bounded variation. One has the inequality

$$
\left|\int_{a}^{b} f(t) d t-\frac{f(a)+f(b)}{2}(b-a)\right| \leq \frac{1}{2}(b-a) \bigvee_{a}^{b}(f),
$$


where $\vee_{a}^{b}(f)$ denotes the total variation of $f$ on the interval $[a, b]$. The constant $1 / 2$ is the best possible one. p. 76]).

This result may be improved if one assumes the monotonicity of $f$ as follows (see [1,

Theorem 1.2. Let $f:[a, b] \rightarrow \mathbb{R}$ be a monotonic nondecreasing function on $[a, b]$. Then one has the inequalities

$$
\begin{aligned}
\left|\int_{a}^{b} f(t) d t-\frac{f(a)+f(b)}{2}(b-a)\right| & \leq \frac{1}{2}(b-a)[f(b)-f(a)]-\int_{a}^{b} \operatorname{sgn}\left(t-\frac{a+b}{2}\right) f(t) d t \\
& \leq \frac{1}{2}(b-a)[f(b)-f(a)] .
\end{aligned}
$$

The above inequalities are sharp.

If the mapping is Lipschitzian, then the following result holds as well [3] (see also [1, p. 82]).

Theorem 1.3. Let $f:[a, b] \rightarrow \mathbb{C}$ be an L-Lipschitzian function on $[a, b]$, that is, $f$ satisfies the condition

$$
|f(s)-f(t)| \leq L|s-t| \text { for any } s, t \in[a, b](L>0 \text { is given }) \text {. }
$$

Then one has the inequality

$$
\left|\int_{a}^{b} f(t) d t-\frac{f(a)+f(b)}{2}(b-a)\right| \leq \frac{1}{4}(b-a)^{2} L .
$$

The constant $1 / 4$ is best in (1.4).

If we would assume absolute continuity for the function $f$, then the following estimates in terms of the Lebesgue norms of the derivative $f^{\prime}$ hold ([1, p. 93]).

Theorem 1.4. Let $f:[a, b] \rightarrow \mathbb{C}$ be an absolutely continuous function on $[a, b]$. Then one has

$$
\begin{aligned}
& \left|\int_{a}^{b} f(t) d t-\frac{f(a)+f(b)}{2}(b-a)\right| \\
& \quad \leq \begin{cases}\frac{1}{4}(b-a)^{2}\left\|f^{\prime}\right\|_{\infty} & \text { if } f^{\prime} \in L_{\infty}[a, b], \\
\frac{1}{2(q+1)^{1 / q}}(b-a)^{1+1 / q}\left\|f^{\prime}\right\|_{p} & \text { if } f^{\prime} \in L_{p}[a, b], p>1, \frac{1}{p}+\frac{1}{q}=1, \\
\frac{1}{2}(b-a)\left\|f^{\prime}\right\|_{1^{\prime}}\end{cases}
\end{aligned}
$$


where $\|\cdot\|_{p}(p \in[1, \infty])$ are the Lebesgue norms, that is,

$$
\begin{gathered}
\left\|f^{\prime}\right\|_{\infty}=\underset{s \in[a, b]}{\operatorname{ess} \sup _{s \in b}\left|f^{\prime}(s)\right|,} \\
\left\|f^{\prime}\right\|_{p}:=\left(\int_{a}^{b}\left|f^{\prime}(s)\right| d s\right)^{1 / p}, \quad p \geq 1 .
\end{gathered}
$$

The case of convex functions is as follows [4].

Theorem 1.5. Let $f:[a, b] \rightarrow \mathbb{R}$ be a convex function on $[a, b]$. Then one has the inequalities

$$
\begin{aligned}
\frac{1}{8}(b-a)^{2}\left[f_{+}^{\prime}\left(\frac{a+b}{2}\right)-f_{-}^{\prime}\left(\frac{a+b}{2}\right)\right] & \leq \frac{f(a)+f(b)}{2}(b-a)-\int_{a}^{b} f(t) d t \\
& \leq \frac{1}{8}(b-a)^{2}\left[f_{-}^{\prime}(b)-f_{+}^{\prime}(a)\right] .
\end{aligned}
$$

The constant $1 / 8$ is sharp in both sides of (1.7).

For other scalar trapezoidal type inequalities, see [2].

\section{Trapezoidal Operator Inequalities}

In order to provide some generalizations for functions of selfadjoint operators of the above trapezoidal inequalities, we need some concepts as results as follows.

Let $A$ be a selfadjoint linear operator on a complex Hilbert space $(H ;\langle\cdot, \cdot\rangle)$. The Gelfand map establishes a *-isometrically isomorphism $\Phi$ between the set $C(\operatorname{Sp}(A))$ of all continuous functions defined on the spectrum of $A$, denoted $\operatorname{Sp}(A)$, and the $C^{*}$-algebra $C^{*}(A)$ generated by $A$ and the identity operator $1_{H}$ on $H$ as follows (see for instance [5, page 3]):

For any $f, g \in C(\operatorname{Sp}(A))$ and any $\alpha, \beta \in \mathbb{C}$ we have

(i) $\Phi(\alpha f+\beta g)=\alpha \Phi(f)+\beta \Phi(g)$;

(ii) $\Phi(f g)=\Phi(f) \Phi(g)$ and $\Phi(\bar{f})=\Phi(f)^{*}$;

(iii) $\|\Phi(f)\|=\|f\|:=\sup _{t \in \operatorname{Sp}(A)}|f(t)|$;

(iv) $\Phi\left(f_{0}\right)=1_{H}$ and $\Phi\left(f_{1}\right)=A$, where $f_{0}(t)=1$ and $f_{1}(t)=t$, for $t \in \operatorname{Sp}(A)$.

With this notation we define

$$
f(A):=\Phi(f) \quad \forall f \in C(\operatorname{Sp}(A))
$$

and we call it the continuous functional calculus for a selfadjoint operator $A$.

If $A$ is a selfadjoint operator and $f$ is a real-valued continuous function on $\operatorname{Sp}(A)$, then $f(t) \geq 0$ for any $t \in \operatorname{Sp}(A)$ implies that $f(A) \geq 0$, for example $f(A)$ is a positive operator on $H$. Moreover, if both $f$ and $g$ are real-valued functions on $\operatorname{Sp}(A)$ then the following important property holds:

$$
f(t) \geq g(t) \text { for any } t \in \operatorname{Sp}(A) \text { implies that } f(A) \geq g(A)
$$

in the operator order of $B(H)$. 
For a recent monograph devoted to various inequalities for continuous functions of selfadjoint operators, see [5] and the references therein.

For other recent results see [6-12].

Let $U$ be a selfadjoint operator on the complex Hilbert space $(H,\langle\cdot, \cdot\rangle)$ with the spectrum $\operatorname{Sp}(U)$ included in the interval $[m, M]$ for some real numbers $m<M$ and let $\left\{E_{\lambda}\right\}_{\lambda}$ be its spectral family. Then for any continuous function $f:[m, M] \rightarrow \mathbb{C}$, it is well-known that we have the following spectral representation in terms of the Riemann-Stieltjes integral:

$$
\langle f(U) x, y\rangle=\int_{m-0}^{M} f(\lambda) d\left(\left\langle E_{\curlywedge} x, y\right\rangle\right)
$$

for any $x, y \in H$. The function $g_{x, y}(\lambda):=\left\langle E_{\lambda} x, y\right\rangle$ is of bounded variation on the interval $[m, M]$ and

$$
g_{x, y}(m-0)=0, \quad g_{x, y}(M)=\langle x, y\rangle
$$

for any $x, y \in H$. It is also well-known that $g_{x}(\lambda):=\left\langle E_{\lambda} x, x\right\rangle$ is monotonic nondecreasing and right continuous on $[m, M]$.

With the notations introduced above, we consider in this paper the problem of bounding the error

$$
\frac{f(M)+f(m)}{2} \cdot\langle x, y\rangle-\langle f(A) x, y\rangle
$$

in approximating $\langle f(A) x, y\rangle$ by the trapezoidal type formula $((f(M)+f(m)) / 2) \cdot\langle x, y\rangle$, where $x, y$ are vectors in the Hilbert space $H, f$ is a continuous functions of the selfadjoint operator $A$ with the spectrum in the compact interval of real numbers $[m, M]$. Applications for some particular elementary functions are also provided.

\section{Some Trapezoidal Vector Inequalities}

The following result holds.

Theorem 3.1. Let $A$ be a selfadjoint operator in the Hilbert space $H$ with the spectrum $\operatorname{Sp}(A) \subseteq$ $[m, M]$ for some real numbers $m<M$ and let $\left\{E_{\lambda}\right\}_{\lambda}$ be its spectral family. If $f:[m, M] \rightarrow \mathbb{C}$ is a continuous function of bounded variation on $[m, M]$, then one has the inequality

$$
\begin{aligned}
& \left|\frac{f(M)+f(m)}{2} \cdot\langle x, y\rangle-\langle f(A) x, y\rangle\right| \\
& \quad \leq \frac{1}{2} \max _{\lambda \in[m, M]}\left[\left\langle E_{\curlywedge} x, x\right\rangle^{1 / 2}\left\langle E_{\curlywedge} y, y\right\rangle^{1 / 2}+\left\langle\left(1_{H}-E_{\curlywedge}\right) x, x\right\rangle^{1 / 2}\left\langle\left(1_{H}-E_{\curlywedge}\right) y, y\right\rangle^{1 / 2}\right] \bigvee_{m}^{M}(f) \\
& \quad \leq \frac{1}{2}\|x\|\|y\|_{m}^{M}(f)
\end{aligned}
$$

for any $x, y \in H$. 
Proof. If $f, u:[m, M] \rightarrow \mathbb{C}$ are such that the Riemann-Stieltjes integral $\int_{a}^{b} f(t) d u(t)$ exists, then a simple integration by parts reveals the identity

$$
\int_{a}^{b} f(t) d u(t)=\frac{f(a)+f(b)}{2}[u(b)-u(a)]-\int_{a}^{b}\left[u(t)-\frac{u(a)+u(b)}{2}\right] d f(t) .
$$

If we write the identity (3.2) for $u(\lambda)=\left\langle E_{\lambda} x, y\right\rangle$, then we get

$$
\int_{m-0}^{M} f(\lambda) d\left(\left\langle E_{\lambda} x, y\right\rangle\right)=\frac{f(m)+f(M)}{2} \cdot\langle x, y\rangle-\int_{m-0}^{M}\left(\left\langle E_{\lambda} x, y\right\rangle-\frac{1}{2}\langle x, y\rangle\right) d f(\lambda)
$$

which, by (2.2), gives the following identity of interest in itself

$$
\frac{f(m)+f(M)}{2} \cdot\langle x, y\rangle-\langle f(A) x, y\rangle=\frac{1}{2} \int_{m-0}^{M}\left[\left\langle E_{\lambda} x, y\right\rangle+\left\langle\left(E_{\curlywedge}-1_{H}\right) x, y\right\rangle\right] d f(\lambda),
$$

for any $x, y \in H$.

It is well-known that if $p:[a, b] \rightarrow \mathbb{C}$ is a continuous function and $v:[a, b] \rightarrow \mathbb{C}$ is of bounded variation, then the Riemann-Stieltjes integral $\int_{a}^{b} p(t) d v(t)$ exists and the following inequality holds:

$$
\left|\int_{a}^{b} p(t) d v(t)\right| \leq \max _{t \in[a, b]}|p(t)| \bigvee_{a}^{b}(v)
$$

where $\vee_{a}^{b}(v)$ denotes the total variation of $v$ on $[a, b]$.

Utilizing the property (3.5), we have from (3.4) that

$$
\begin{aligned}
\left|\frac{f(m)+f(M)}{2} \cdot\langle x, y\rangle-\langle f(A) x, y\rangle\right| & \leq \frac{1}{2} \max _{\lambda \in[m, M]}\left|\left\langle E_{\lambda} x, y\right\rangle+\left\langle\left(E_{\lambda}-1_{H}\right) x, y\right\rangle\right| \bigvee_{m}^{M}(f) \\
& \leq \frac{1}{2}\left[\max _{\lambda \in[m, M]}\left[\left|\left\langle E_{\lambda} x, y\right\rangle\right|+\left|\left\langle\left(1_{H}-E_{\curlywedge}\right) x, y\right\rangle\right|\right]\right] \bigvee_{m}^{M}(f)
\end{aligned}
$$

If $P$ is a nonnegative operator on $H$, that is, $\langle P x, x\rangle \geq 0$ for any $x \in H$, then the following inequality is a generalization of the Schwarz inequality in the Hilbert space $H$ :

$$
|\langle P x, y\rangle|^{2} \leq\langle P x, x\rangle\langle P y, y\rangle
$$

for any $x, y \in H$. 
On applying the inequality (3.7) we have

$$
\begin{gathered}
\left|\left\langle E_{\lambda} x, y\right\rangle\right| \leq\left\langle E_{\curlywedge} x, x\right\rangle^{1 / 2}\left\langle E_{\lambda} y, y\right\rangle^{1 / 2}, \\
\left|\left\langle\left(1_{H}-E_{\curlywedge}\right) x, y\right\rangle\right| \leq\left\langle\left(1_{H}-E_{\curlywedge}\right) x, x\right\rangle^{1 / 2}\left\langle\left(1_{H}-E_{\curlywedge}\right) y, y\right\rangle^{1 / 2},
\end{gathered}
$$

which, together with the elementary inequality for $a, b, c, d \geq 0$

$$
a b+c d \leq\left(a^{2}+c^{2}\right)^{1 / 2}\left(b^{2}+d^{2}\right)^{1 / 2}
$$

produce the inequalities

$$
\begin{aligned}
& \left|\left\langle E_{\lambda} x, y\right\rangle\right|+\left|\left\langle\left(1_{H}-E_{\curlywedge}\right) x, y\right\rangle\right| \\
& \quad \leq\left\langle E_{\curlywedge} x, x\right\rangle^{1 / 2}\left\langle E_{\curlywedge} y, y\right\rangle^{1 / 2}+\left\langle\left(1_{H}-E_{\curlywedge}\right) x, x\right\rangle^{1 / 2}\left\langle\left(1_{H}-E_{\curlywedge}\right) y, y\right\rangle^{1 / 2} \\
& \quad \leq\left(\left\langle E_{\lambda} x, x\right\rangle+\left\langle\left(1_{H}-E_{\curlywedge}\right) x, x\right\rangle\right)^{1 / 2}\left(\left\langle E_{\curlywedge} y, y\right\rangle+\left\langle\left(1_{H}-E_{\curlywedge}\right) y, y\right\rangle\right)^{1 / 2} \\
& \quad=\|x\|\|y\|
\end{aligned}
$$

for any $x, y \in H$.

On utilizing (3.6) and taking the maximum in (3.10) we deduce the desired result

The case of Lipschitzian functions may be useful for applications.

Theorem 3.2. Let $A$ be a selfadjoint operator in the Hilbert space $H$ with the spectrum $\operatorname{Sp}(A) \subseteq$ $[m, M]$ for some real numbers $m<M$ and let $\left\{E_{\lambda}\right\}_{\curlywedge}$ be its spectral family. If $f:[m, M] \rightarrow \mathbb{C}$ is Lipschitzian with the constant $L>0$ on $[m, M]$, then one has the inequality

$$
\begin{aligned}
& \left|\frac{f(M)+f(m)}{2} \cdot\langle x, y\rangle-\langle f(A) x, y\rangle\right| \\
& \quad \leq \frac{1}{2} L \int_{m-0}^{M}\left[\left\langle E_{\curlywedge} x, x\right\rangle^{1 / 2}\left\langle E_{\curlywedge} y, y\right\rangle^{1 / 2}+\left\langle\left(1_{H}-E_{\curlywedge}\right) x, x\right\rangle^{1 / 2}\left\langle\left(1_{H}-E_{\curlywedge}\right) y, y\right\rangle^{1 / 2}\right] d \lambda \\
& \quad \leq \frac{1}{2}(M-m) L\|x\|\|y\|
\end{aligned}
$$

for any $x, y \in H$. 
Proof. It is well-known that if $p:[a, b] \rightarrow \mathbb{C}$ is a Riemann integrable function and $v:[a, b] \rightarrow$ $\mathbb{C}$ is Lipschitzian with the constant $L>0$, that is,

$$
|v(s)-v(t)| \leq L|s-t| \text { for any } t, s \in[a, b]
$$

then the Riemann-Stieltjes integral $\int_{a}^{b} p(t) d v(t)$ exists and the following inequality holds:

$$
\left|\int_{a}^{b} p(t) d v(t)\right| \leq L \int_{a}^{b}|p(t)| d t .
$$

Now, on applying this property of the Riemann-Stieltjes integral, we have from the representation (3.4) that

$$
\begin{aligned}
\left|\frac{f(m)+f(M)}{2} \cdot\langle x, y\rangle-\langle f(A) x, y\rangle\right| & \leq \frac{1}{2} L \int_{m-0}^{M}\left|\left\langle E_{\lambda} x, y\right\rangle+\left\langle\left(E_{\curlywedge}-1_{H}\right) x, y\right\rangle\right| d \lambda \\
& \leq \frac{1}{2} L \int_{m-0}^{M}\left[\left|\left\langle E_{\curlywedge} x, y\right\rangle\right|+\left|\left\langle\left(1_{H}-E_{\curlywedge}\right) x, y\right\rangle\right|\right] d \lambda
\end{aligned}
$$

for any $x, y \in H$.

Further, integrating (3.10) on $[m, M]$ we have

$$
\begin{aligned}
& \int_{m-0}^{M}\left[\left|\left\langle E_{\lambda} x, y\right\rangle\right|+\left|\left\langle\left(1_{H}-E_{\curlywedge}\right) x, y\right\rangle\right|\right] d \lambda \\
& \quad \leq \int_{m-0}^{M}\left[\left\langle E_{\curlywedge} x, x\right\rangle^{1 / 2}\left\langle E_{\curlywedge} y, y\right\rangle^{1 / 2}+\left\langle\left(1_{H}-E_{\curlywedge}\right) x, x\right\rangle^{1 / 2}\left\langle\left(1_{H}-E_{\curlywedge}\right) y, y\right\rangle^{1 / 2}\right] d \lambda \\
& \quad \leq(M-m)\|x\|\|y\|
\end{aligned}
$$

which together with (3.14) produces the desired result (3.11).

\section{Other Trapezoidal Vector Inequalities}

The following result provides a different perspective in bounding the error in the trapezoidal approximation. 
Theorem 4.1. Let $A$ be a selfadjoint operator in the Hilbert space $H$ with the spectrum $\operatorname{Sp}(A) \subseteq$ $[m, M]$ for some real numbers $m<M$ and let $\left\{E_{\lambda}\right\}_{\lambda}$ be its spectral family. Assume that $f$ : $[m, M] \rightarrow \mathbb{C}$ is a continuous function on $[m, M]$. Then one has the inequalities

$$
\begin{aligned}
& \left|\frac{f(M)+f(m)}{2} \cdot\langle x, y\rangle-\langle f(A) x, y\rangle\right| \\
& \int \max _{\lambda \in[m, M]}\left|\left\langle E_{\lambda} x-\frac{1}{2} x, y\right\rangle\right| \bigvee_{m}^{M}(f) \text { if } f \text { is of bounded variation, } \\
& \leq\left\{\begin{array}{l}
L \int_{m-0}^{M}\left|\left\langle E_{\lambda} x-\frac{1}{2} x, y\right\rangle\right| d \lambda \quad \text { if } f \text { is } L \text { Lipschitzian, } \\
\int_{m-0}^{M}\left|\left\langle E_{\lambda} x-\frac{1}{2} x, y\right\rangle\right| d f(\lambda) \quad \text { if } f \text { is nondecreasing, }
\end{array}\right. \\
& \leq \frac{1}{2}\|x\|\|y\|\left\{\begin{array}{l}
\bigvee_{m}^{M}(f) \quad \text { if } f \text { is of bounded variation, } \\
L(M-m) \quad \text { if } f \text { is } L \text { Lipschitzian, } \\
(f(M)-f(m)) \text { if } f \text { is nondecreasing }
\end{array}\right.
\end{aligned}
$$

for any $x, y \in H$.

Proof. From (3.6) we have that

$$
\begin{aligned}
\left|\frac{f(m)+f(M)}{2} \cdot\langle x, y\rangle-\langle f(A) x, y\rangle\right| & \leq \frac{1}{2} \max _{\lambda \in[m, M]}\left|\left\langle E_{\curlywedge} x, y\right\rangle+\left\langle\left(E_{\curlywedge}-1_{H}\right) x, y\right\rangle\right| \bigvee_{m}^{M}(f) \\
& =\max _{\lambda \in[m, M]}\left|\left\langle E_{\curlywedge} x-\frac{1}{2} x, y\right\rangle\right| \bigvee_{m}^{M}(f)
\end{aligned}
$$

for any $x, y \in H$.

Utilizing the Schwarz inequality in $H$ and the fact that $E_{\mathcal{\lambda}}$ are projectors we have successively

$$
\begin{aligned}
\left|\left\langle E_{\curlywedge} x-\frac{1}{2} x, y\right\rangle\right| & \leq\left\|E_{\curlywedge} x-\frac{1}{2} x\right\|\|y\| \\
& =\left[\left\langle E_{\curlywedge} x, E_{\curlywedge} x\right\rangle-\left\langle E_{\curlywedge} x, x\right\rangle+\frac{1}{4}\|x\|^{2}\right]^{1 / 2}\|y\| \\
& =\frac{1}{2}\|x\|\|y\|
\end{aligned}
$$

for any $x, y \in H$, which proves the first branch in (4.1).

The second inequality follows from (3.14). 
From the theory of Riemann-Stieltjes integral is well-known that if $p:[a, b] \rightarrow \mathbb{C}$ is of bounded variation and $v:[a, b] \rightarrow \mathbb{R}$ is continuous and monotonic nondecreasing, then the Riemann-Stieltjes integrals $\int_{a}^{b} p(t) d v(t)$ and $\int_{a}^{b}|p(t)| d v(t)$ exist and

$$
\left|\int_{a}^{b} p(t) d v(t)\right| \leq \int_{a}^{b}|p(t)| d v(t)
$$

From the representation (3.4) we then have

$$
\begin{aligned}
\left|\frac{f(m)+f(M)}{2} \cdot\langle x, y\rangle-\langle f(A) x, y\rangle\right| & \leq \frac{1}{2} \int_{m-0}^{M}\left|\left\langle E_{\lambda} x, y\right\rangle+\left\langle\left(E_{\lambda}-1_{H}\right) x, y\right\rangle\right| d f(\lambda) \\
& =\int_{m-0}^{M}\left|\left\langle E_{\lambda} x-\frac{1}{2} x, y\right\rangle\right| d f(\lambda)
\end{aligned}
$$

for any $x, y \in H$, from which we obtain the last branch in (4.1).

We recall that a function $f:[a, b] \rightarrow \mathbb{C}$ is called $r$-H-Hölder continuous with fixed $r \in(0,1]$ and $H>0$ if

$$
|f(t)-f(s)| \leq H|t-s|^{r} \quad \text { for any } t, s \in[a, b] .
$$

We have the following result concerning this class of functions.

Theorem 4.2. Let $A$ be a selfadjoint operator in the Hilbert space $H$ with the spectrum $\operatorname{Sp}(A) \subseteq$ $[m, M]$ for some real numbers $m<M$ and let $\left\{E_{\lambda}\right\}_{\lambda}$ be its spectral family. If $f:[m, M] \rightarrow \mathbb{C}$ is $r-H$-Hölder continuous on $[m, M]$, then one has the inequality

$$
\begin{aligned}
\left|\frac{f(m)+f(M)}{2} \cdot\langle x, y\rangle-\langle f(A) x, y\rangle\right| & \leq \frac{1}{2^{r}} H(M-m)^{r} \bigvee_{m}^{M}\left(\left\langle E_{(\cdot)} x, y\right\rangle\right) \\
& \leq \frac{1}{2^{r}} H(M-m)^{r}\|x\|\|y\|
\end{aligned}
$$

for any $x, y \in H$.

Proof. We start with the equality

$$
\frac{f(M)+f(m)}{2} \cdot\langle x, y\rangle-\langle f(A) x, y\rangle=\int_{m-0}^{M}\left[\frac{f(M)+f(m)}{2}-f(\lambda)\right] d\left(\left\langle E_{\lambda} x, y\right\rangle\right)
$$

for any $x, y \in H$, that follows from the spectral representation (2.2). 
Since the function $\left\langle E_{(\cdot)} x, y\right\rangle$ is of bounded variation for any vector $x, y \in H$, by applying the inequality (3.5) we conclude that

$$
\left|\frac{f(m)+f(M)}{2} \cdot\langle x, y\rangle-\langle f(A) x, y\rangle\right| \leq \max _{\lambda \in[m, M]}\left|\frac{f(M)+f(m)}{2}-f(\lambda)\right| \bigvee_{m}^{M}\left(\left\langle E_{(\cdot)} x, y\right\rangle\right)
$$

for any $x, y \in H$.

As $f:[m, M] \rightarrow \mathbb{C}$ is $r$-H-Hölder continuous on $[m, M]$, then we have

$$
\begin{aligned}
\left|\frac{f(M)+f(m)}{2}-f(\lambda)\right| & \leq \frac{1}{2}|f(M)-f(\lambda)|+\frac{1}{2}|f(\lambda)-f(m)| \\
& \leq \frac{1}{2} H\left[(M-\lambda)^{r}+(\lambda-m)^{r}\right]
\end{aligned}
$$

for any $\lambda \in[m, M]$.

Since, obviously, the function $g_{r}(\lambda):=(M-\lambda)^{r}+(\lambda-m)^{r}, r \in(0,1]$ has the property that

$$
\max _{\lambda \in[m, M]} g_{r}(\lambda)=g_{r}\left(\frac{m+M}{2}\right)=2^{1-r}(M-m)^{r},
$$

then by (4.9) we deduce the first part of (4.7).

Now, if $d: m=t_{0}<t_{1}<\cdots<t_{n-1}<t_{n}=M$ is an arbitrary partition of the interval $[m, M]$, then we have by the Schwarz inequality for nonnegative operators that

$$
\begin{aligned}
\bigvee_{m}^{M}\left(\left\langle E_{(\cdot)} x, y\right\rangle\right) & =\sup _{d}\left\{\sum_{i=0}^{n-1}\left|\left\langle\left(E_{t_{i+1}}-E_{t_{i}}\right) x, y\right\rangle\right|\right\} \\
& \leq \sup _{d}\left\{\sum_{i=0}^{n-1}\left[\left\langle\left(E_{t_{i+1}}-E_{t_{i}}\right) x, x\right\rangle^{1 / 2}\left\langle\left(E_{t_{i+1}}-E_{t_{i}}\right) y, y\right\rangle^{1 / 2}\right]\right\}:=I .
\end{aligned}
$$

By the Cauchy-Buniakovski-Schwarz inequality for sequences of real numbers we also have that

$$
\begin{aligned}
I & \leq \sup _{d}\left\{\left[\sum_{i=0}^{n-1}\left\langle\left(E_{t_{i+1}}-E_{t_{i}}\right) x, x\right\rangle\right]^{1 / 2}\left[\sum_{i=0}^{n-1}\left\langle\left(E_{t_{i+1}}-E_{t_{i}}\right) y, y\right\rangle\right]^{1 / 2}\right\} \\
& \leq \sup _{d}\left\{\left[\sum_{i=0}^{n-1}\left\langle\left(E_{t_{i+1}}-E_{t_{i}}\right) x, x\right\rangle\right]^{1 / 2} \sup _{d}\left[\sum_{i=0}^{n-1}\left\langle\left(E_{t_{i+1}}-E_{t_{i}}\right) y, y\right\rangle\right]^{1 / 2}\right\} \\
& =\left[\bigvee_{m}^{M}\left(\left\langle E_{(\cdot)} x, x\right\rangle\right)\right]^{1 / 2}\left[\bigvee_{m}^{M}\left(\left\langle E_{(\cdot)} y, y\right\rangle\right)\right]^{1 / 2}=\|x\|\|y\|
\end{aligned}
$$

for any $x, y \in H$. These prove the last part of (4.7). 


\section{Applications for Some Particular Functions}

It is obvious that the results established above can be applied for various particular functions of selfadjoint operators. We restrict ourselves here to only two examples, namely, the logarithm and the power functions.

(1) If we consider the logarithmic function $f:(0, \infty) \rightarrow \mathbb{R}, f(t)=\ln t$, then we can state the following result.

Proposition 5.1. Let $A$ be a selfadjoint operator in the Hilbert space $H$ with the spectrum $\operatorname{Sp}(A) \subseteq$ $[m, M]$ for some real numbers with $0<m<M$ and let $\left\{E_{\lambda}\right\}_{\lambda}$ be its spectral family. Then for any $x, y \in H$ one has

$$
\begin{aligned}
& |\langle x, y\rangle \ln \sqrt{m M}-\langle\ln A x, y\rangle| \\
& \quad \leq \ln \left(\frac{M}{m}\right) \times\left\{\begin{array}{l}
\frac{1}{2} \max _{\lambda \in[m, M]}\left[\left\langle E_{\lambda} x, x\right\rangle^{1 / 2}\left\langle E_{\curlywedge} y, y\right\rangle^{1 / 2}+\left\langle\left(1_{H}-E_{\curlywedge}\right) x, x\right\rangle^{1 / 2}\left\langle\left(1_{H}-E_{\curlywedge}\right) y, y\right\rangle^{1 / 2}\right] \\
\max _{\lambda \in[m, M]}\left|\left\langle E_{\lambda} x-\frac{1}{2} x, y\right\rangle\right|
\end{array}\right. \\
& \quad \leq \frac{1}{2}\|x\|\|y\| \ln \left(\frac{M}{m}\right), \\
& |\langle x, y\rangle \ln \sqrt{m M}-\langle\ln A x, y\rangle| \\
& \quad \leq \frac{1}{m} \times\left\{\begin{array}{l}
\frac{1}{2} \int_{m-0}^{M}\left[\left\langle E_{\lambda} x, x\right\rangle^{1 / 2}\left\langle E_{\lambda} y, y\right\rangle^{1 / 2}+\left\langle\left(1_{H}-E_{\curlywedge}\right) x, x\right\rangle^{1 / 2}\left\langle\left(1_{H}-E_{\curlywedge}\right) y, y\right\rangle^{1 / 2}\right] d \lambda \\
\int_{m-0}^{M}\left|\left\langle E_{\lambda} x-\frac{1}{2} x, y\right\rangle\right| d \lambda
\end{array}\right. \\
& \quad \leq \frac{1}{2}\|x\|\|y\|\left(\frac{M}{m}-1\right), \\
& \quad \leq \frac{1}{2}\|x\|\|y\| \ln \left(\frac{M}{m}\right) . \\
& |\langle x, y\rangle \ln \sqrt{m M}-\langle\ln A x, y\rangle| \leq \int_{m-0}^{M}\left|\left\langle E_{\curlywedge} x-\frac{1}{2} x, y\right\rangle\right| \lambda^{-1} d \lambda
\end{aligned}
$$

The proof is obvious from Theorems 3.1, 3.2, and 4.1 applied for the logarithmic function. The details are omitted.

(2) Consider now the power function $f:(0, \infty) \rightarrow \mathbb{R}, f(t)=t^{p}$ with $p \in(-\infty, 0) \cup$ $(0, \infty)$. In the case when $p \in(0,1)$, the function is $p$-H-Hölder continuous with $H=1$ on any subinterval $[m, M]$ of $[0, \infty)$. By making use of Theorem 4.2 we can state the following result. 
Proposition 5.2. Let $A$ be a selfadjoint operator in the Hilbert space $H$ with the spectrum $\operatorname{Sp}(A) \subseteq$ $[m, M]$ for some real numbers with $0 \leq m<M$ and let $\left\{E_{\lambda}\right\}_{\lambda}$ be its spectral family. Then for $p \in(0,1)$ one has

$$
\begin{aligned}
\left|\frac{m^{p}+M^{p}}{2} \cdot\langle x, y\rangle-\left\langle A^{p} x, y\right\rangle\right| & \leq \frac{1}{2^{p}}(M-m)^{p} \bigvee_{m}^{M}\left(\left\langle E_{(\cdot)} x, y\right\rangle\right) \\
& \leq \frac{1}{2^{p}}(M-m)^{p}\|x\|\|y\|,
\end{aligned}
$$

for any $x, y \in H$.

The case of powers $p \geq 1$ is embodied in the following.

Proposition 5.3. Let $A$ be a selfadjoint operator in the Hilbert space $H$ with the spectrum $\operatorname{Sp}(A) \subseteq$ $[m, M]$ for some real numbers with $0 \leq m<M$ and let $\left\{E_{\lambda}\right\}_{\lambda}$ be its spectral family. Then for $p \geq 1$ and for any $x, y \in H$ one has

$$
\begin{aligned}
& \left|\frac{m^{p}+M^{p}}{2} \cdot\langle x, y\rangle-\left\langle A^{p} x, y\right\rangle\right| \\
& \quad \leq\left(M^{p}-m^{p}\right) \times\left\{\begin{array}{l}
\frac{1}{2} \max _{\lambda \in[m, M]}\left[\left\langle E_{\curlywedge} x, x\right\rangle^{1 / 2}\left\langle E_{\lambda} y, y\right\rangle^{1 / 2}\right. \\
\left.+\left\langle\left(1_{H}-E_{\curlywedge}\right) x, x\right\rangle^{1 / 2}\left\langle\left(1_{H}-E_{\lambda}\right) y, y\right\rangle^{1 / 2}\right] \\
\max _{\lambda \in[m, M]}\left|\left\langle E_{\lambda} x-\frac{1}{2} x, y\right\rangle\right|
\end{array}\right. \\
& \leq \frac{1}{2}\|x\|\|y\|\left(M^{p}-m^{p}\right),
\end{aligned}
$$

$$
\begin{aligned}
& \left|\frac{m^{p}+M^{p}}{2} \cdot\langle x, y\rangle-\left\langle A^{p} x, y\right\rangle\right| \\
& \quad \leq p M^{p-1} \times\left\{\begin{array}{l}
\frac{1}{2} \int_{m-0}^{M}\left[\left\langle E_{\curlywedge} x, x\right\rangle^{1 / 2}\left\langle E_{\curlywedge} y, y\right\rangle^{1 / 2}+\left\langle\left(1_{H}-E_{\curlywedge}\right) x, x\right\rangle^{1 / 2}\left\langle\left(1_{H}-E_{\curlywedge}\right) y, y\right\rangle^{1 / 2}\right] d \lambda \\
\int_{m-0}^{M}\left|\left\langle E_{\curlywedge} x-\frac{1}{2} x, y\right\rangle\right| d \lambda
\end{array}\right. \\
& \quad \leq \frac{1}{2} p\|x\|\|y\| M^{p-1}, \\
& \quad \begin{array}{c}
\frac{m^{p}+M^{p}}{2} \cdot\langle x, y\rangle-\left\langle A^{p} x, y\right\rangle\left|\leq p \int_{m-0}^{M}\right|\left\langle E_{\curlywedge} x-\frac{1}{2} x, y\right\rangle \mid \lambda^{p-1} d \lambda \\
\quad \leq \frac{1}{2}\|x\| y \|\left(M^{p}-m^{p}\right) .
\end{array}
\end{aligned}
$$


The proof is obvious from Theorems 3.1, 3.2, and 4.1 applied for the power function $f:(0, \infty) \rightarrow \mathbb{R}, f(t)=t^{p}$ with $p \geq 1$. The details are omitted.

The case of negative powers is similar. The details are left to the interested reader.

\section{Acknowledgment}

The author would like to thank the anonymous referees for their valuable comments that have been implemented in the final version of the paper.

\section{References}

[1] P. Cerone, S. S. Dragomir, and C. E. M. Pearce, "A generalized trapezoid inequality for functions of bounded variation," Turkish Journal of Mathematics, vol. 24, no. 2, pp. 147-163, 2000.

[2] P. Cerone and S. S. Dragomir, "Trapezoidal-type rules from an inequalities point of view," in Handbook of Analytic-Computational Methods in Applied Mathematics, pp. 65-134, Chapman \& Hall/CRC, New York, NY, USA, 2000.

[3] S. S. Dragomir, "On the trapezoid quadrature formula for Lipschitzian mappings and applications," Tamkang Journal of Mathematics, vol. 30, no. 2, pp. 133-138, 1999.

[4] S. S. Dragomir, "An inequality improving the second Hermite-Hadamard inequality for convex functions defined on linear spaces and applications for semi-inner products," Journal of Inequalities in Pure and Applied Mathematics, vol. 3, no. 3, article 2002, 2002.

[5] T. Furuta, J. Mićić Hot, J. Pečarić, and Y. Seo, Mond-Pečarić Method in Operator Inequalities. Inequalities for Bounded Selfadjoint Operators on a Hilbert Space, Element, Zagreb, Croatia, 2005.

[6] S. S. Dragomir, "Čebyšev's type inequalities for functions of selfadjoint operators in Hilbert spaces," Research Group in Mathematical Inequalities and Applications, vol. 11, article 9, 2008.

[7] S. S. Dragomir, "Grüss' type inequalities for functions of selfadjoint operators in Hilbert spaces," Research Group in Mathematical Inequalities and Applications, vol. 11, article 11, 2008.

[8] S. S. Dragomir, "Inequalities for the Čebyšev functional of two functions of selfadjoint operators in Hilbert spaces," Research Group in Mathematical Inequalities and Applications, vol. 11, 2008.

[9] A. Matković, J. Pečarić, and I. Perić, "A variant of Jensen's inequality of Mercer's type for operators with applications," Linear Algebra and Its Applications, vol. 418, no. 2-3, pp. 551-564, 2006.

[10] B. Mond and J. E. Pečarić, "Convex inequalities in Hilbert space," Houston Journal of Mathematics, vol. 19, no. 3, pp. 405-420, 1993.

[11] B. Mond and J. E. Pečarić, “Classical inequalities for matrix functions," Utilitas Mathematica, vol. 46, pp. 155-166, 1994.

[12] J. Pečarić, J. Mićić, and Y. Seo, "Inequalities between operator means based on the Mond-Pečarić method," Houston Journal of Mathematics, vol. 30, no. 1, pp. 191-207, 2004. 


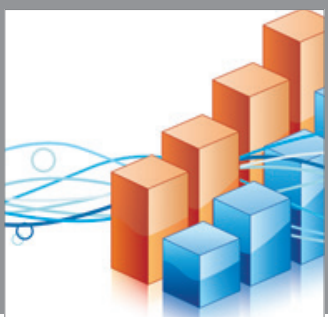

Advances in

Operations Research

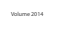

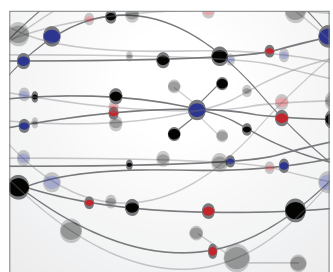

\section{The Scientific} World Journal
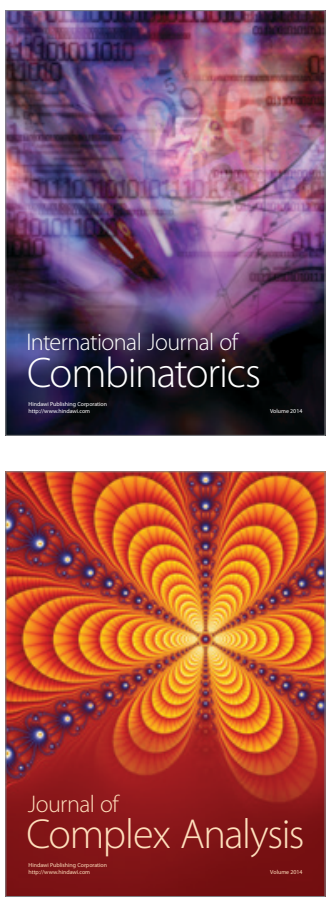

International Journal of

Mathematics and

Mathematical

Sciences
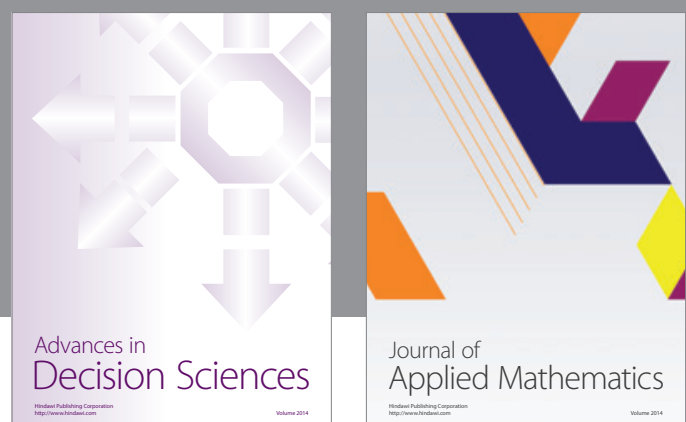

Journal of

Applied Mathematics
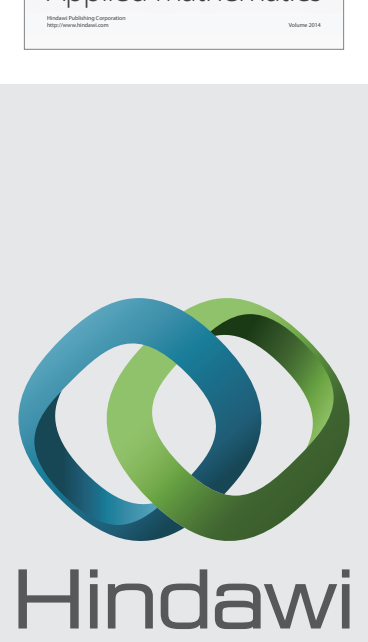

Submit your manuscripts at http://www.hindawi.com
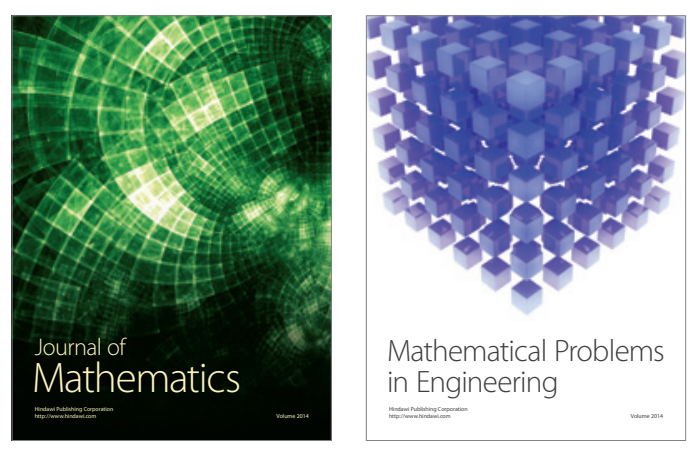

Mathematical Problems in Engineering
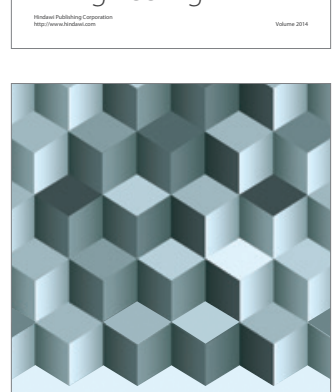

Journal of

Function Spaces
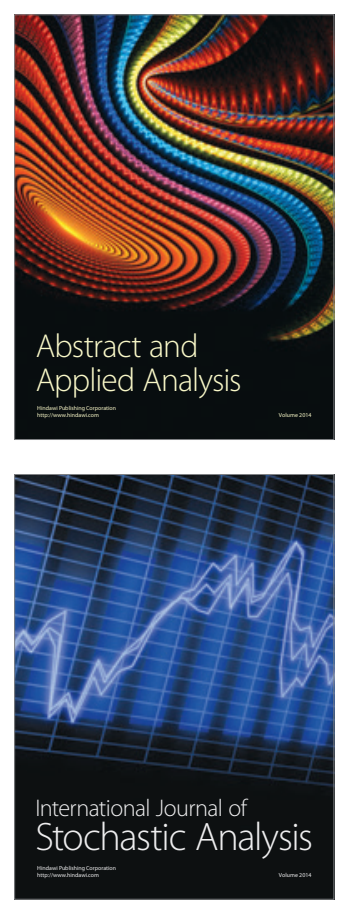

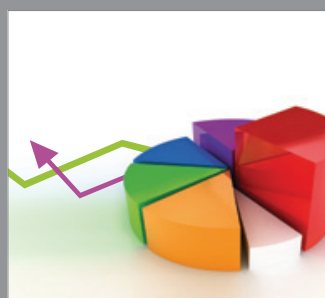

ournal of

Probability and Statistics

Promensencen
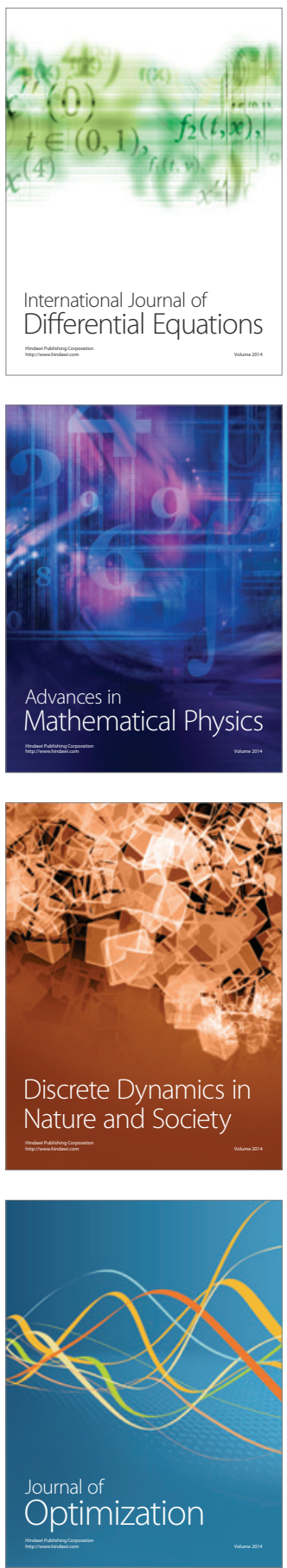